Article

\title{
Effects of Dietary Forage Proportion on Feed Intake, Growth Performance, Nutrient Digestibility, and Enteric Methane Emissions of Holstein Heifers at Various Growth Stages
}

\author{
Lifeng Dong, Binchang Li and Qiyu Diao * (B) \\ Feed Research Institute, Chinese Academy of Agricultural Sciences/Beijing Key Laboratory for Dairy Cow \\ Nutrition/Key Laboratory of Feed Biotechnology, Ministry of Agriculture/Sino-US Joint Lab on Nutrition and \\ Metabolism of Ruminant, Beijing 100081, China; donglifeng@email.ulster.ac.uk or donglifeng@caas.cn (L.D.); \\ 118394151270@163.com (B.L.) \\ * Correspondence: diaoqiyu@caas.cn; Tel.: +86-010-82106055
}

Received: 18 July 2019; Accepted: 23 September 2019; Published: 26 September 2019

Simple Summary: Enteric methane $\left(\mathrm{CH}_{4}\right)$ emission from ruminants is a large source of anthropogenic greenhouse gas production, which is an inevitable by-product when feedstuff is digested and fermented in the rumen, representing approximately $7 \%$ of dietary energy loss. Although the Chinese government has committed to reduce $\mathrm{CH}_{4}$ emissions under the requirement of the Copenhagen Accord (2009), there is lack of accurate $\mathrm{CH}_{4}$ emission data from young cows as the guideline of IPCC gives little consideration to the variations of geographic conditions, animal physiology stages, and dietary components of dairy production system. Our study investigated the effects of different dietary forage-to-concentrate on feed intake, growth performance, nutrient digestibility, and enteric $\mathrm{CH}_{4}$ emissions of Holstein heifers under various growth stage, and developed the prediction equations using production and emission data. Our results demonstrated that enteric $\mathrm{CH}_{4}$ emission was significantly affected by dietary composition and physiological condition; results obtained from the current study will be of great importance for development of regional or national emission inventories and mitigation approaches for heifers at specific growth stage.

Abstract: Enteric methane $\left(\mathrm{CH}_{4}\right)$ emissions from young ruminants contribute to a substantial proportion of atmospheric $\mathrm{CH}_{4}$ accumulation. Development of emission inventory and mitigation approaches needs accurate estimation of individual emission from animals under various physiological conditions and production systems. This research investigated the effect of different dietary concentrate contents on feed intake, growth performance, nutrient digestibility and $\mathrm{CH}_{4}$ emissions of heifers at various stages, and also developed linear or non-linear prediction equations using data measured by sulphur hexafluoride tracer technique. Increasing dietary concentrate contents increased feed intake and growth rate, enhanced nutrient digestibility, and reduced enteric $\mathrm{CH}_{4}$ emissions. Heifers at the age of 9,12, and 15 months with an average weight of 267.7, 342.1, and $418.6 \mathrm{~kg}$ produced 105.2, 137.4, and $209.4 \mathrm{~g} /$ day of $\mathrm{CH}_{4}$, and have an average value of $\mathrm{CH}_{4}$ energy per gross energy intake $\left(\mathrm{Y}_{\mathrm{m}}\right)$ 0.054, 0.064, 0.0667, respectively. Equations relating $\mathrm{CH}_{4}$ emission values with animal and feed characteristics were developed with high determination coefficients for heifers at different growth stages. Dietary concentrate contents had significant influence on overall performance of heifers. These data can be used to develop regional or national emission inventories and mitigation approaches for heifers under various production regimes in China.

Keywords: methane; heifer; forage-to-concentrate ratio; prediction equation; sulphur hexafluoride tracer technique 


\section{Introduction}

Enteric methane $\left(\mathrm{CH}_{4}\right)$ is a final product of ruminal fermentation via methanogenesis, which contributes substantially to atmospheric $\mathrm{CH}_{4}$ accumulation. As dietary structural carbohydrates (e.g., cellulose and hemicellulose) are degraded by ruminal microorganisms, $\mathrm{CH}_{4}$ emission represents up to $12 \%$ loss of dietary energy ingested in the rumen [1]. Thus, reducing enteric $\mathrm{CH}_{4}$ emissions will help improve energy utilization efficiency and alleviate environmental pressures for dairy production regimes. According to the national report, $\mathrm{CH}_{4}$ emissions in 2005 from agriculture sector accounts for $25.5 \mathrm{Tg}$, of which approximately $57 \%$ is from rumen fermentation within ruminant production system in China [2]. As dairy population and milk production increased by 24 and 58 times, respectively, from 1961 to 2010, it is projected that the total $\mathrm{CH}_{4}$ emissions in 2030 will reach $52.1 \mathrm{Tg}$ [3]. Meanwhile, in response to the domestic and international pressures on sustainable development, the Chinese government has made the commitment to reduce greenhouse gases (GHG), which has been incorporated in to the 2009 Copenhagen Accord [4]. Although Tier-2 methodology from International Panel on Climate Change (IPCC) guidelines is commonly used in many countries for the quantification of $\mathrm{CH}_{4}$ emission inventories [5], it gives little consideration to the variations of geographic conditions, animal physiology stages, and dietary components [6,7]. In addition, this methodology tries to calculate the $\mathrm{CH}_{4}$ emission for the whole dairy population using a default value derived from lactating cows. In China, approximately $60 \%$ of the dairy population is milking cows, and the remainder is heifers (i.e., 5.68 million of heifers) [8]. Different physiological conditions and varied composition and abundance of ruminal methanogens demonstrated great difference of $\mathrm{CH}_{4}$ emission of lactating cow and heifer, indicating the importance of quantifying the individual emissions for these animals [3]. However, limited studies have examined the effects of dietary components on $\mathrm{CH}_{4}$ emissions of heifers under different physiological conditions. Therefore, the objective of the current study is to assess the effect of different dietary concentrate contents on the enteric $\mathrm{CH}_{4}$ emissions of Holstein heifers at various stage, and develop prediction equations using data collected using sulphur hexafluoride $\left(\mathrm{SF}_{6}\right)$ tracer technique.

\section{Materials and Methods}

\subsection{Animals, Experimental Design, and Diets}

This study was conducted in 2018 at the Zhongjiayonghong dairy farm located in Fangshan district, (Beijing, China, latitude: N39 $39^{\prime} 6^{\prime \prime}$ and longitude: E116 $\left.{ }^{\circ} 12^{\prime} 21^{\prime \prime}\right)$. Forty-five Chinese Holstein heifers with an initial body weight (BW) of $264.9 \pm 25.6 \mathrm{~kg}$ (mean \pm SD) were used in this study with three measurements taken at age of 9,12, and 15 months, respectively. In each experiment stage, heifers were balanced by date of birth, age and BW and offered randomly assigned to 1 of 3 treatments $(n=15)$ in which animals were individually offered diets containing 30,40 , and $50 \%$ of concentrate (C30, C40, and C50, respectively). Each experimental period was 32 days in length, including 18 days for adaptation, followed by 8 days for gas measurement and 6 days for nutrient digestibility. Heifers were housed individually with free access to feed and water throughout the whole experiment. All animal care and handling procedures were reviewed and approved by the Animal Ethics Committee of Chinese Academy of Agricultural Sciences (protocol number 019-2018) prior to the start of the experiment.

In period 1, cows received their diet as a total mixed rations (TMR) that composted of corn silage, wildrye, and a typical ration of concentrate on Chinese commercial farms. In period 2 and 3, alfalfa was included in the diet based on the ration of period 1 (Table 1). The TMR were prepared daily using a feed mixer (Belle Engineering Ltd., Derbyshire, UK) and distributed ad libitum (5\% refusals, on an as-fed basis). All diets in three periods were formulated to meet the recommendation of Ministry of Agriculture of P. R. China. For all of the three periods, cows were fed twice daily between 0600 and $0800 \mathrm{~h}$, and 1600 and $1800 \mathrm{~h}$. Feed refusals were collected and weighted to determine the daily feed intake. 
Table 1. Ingredient and chemical composition of diets in the current study.

\begin{tabular}{|c|c|c|c|c|c|c|}
\hline \multirow{2}{*}{ Item } & \multicolumn{3}{|c|}{ Period 1 (9 months) } & \multicolumn{3}{|c|}{ Periods 2 and 3 (12 and 15 months) } \\
\hline & $\mathrm{C} 30$ & $\mathrm{C} 40$ & $\mathrm{C} 50$ & $\mathrm{C} 30$ & $\mathrm{C} 40$ & $\mathrm{C} 50$ \\
\hline \multicolumn{7}{|l|}{ Ingredient } \\
\hline Corn silage & 42 & 36 & 30 & 42 & 36 & 30 \\
\hline Chinese wildrye hay & 28 & 24 & 20 & 14 & 12 & 10 \\
\hline Alfalfa & - & - & - & 14 & 12 & 10 \\
\hline Concentrate & 30 & 40 & 50 & 30 & 40 & 50 \\
\hline \multicolumn{7}{|l|}{ Nutrient, DM basis } \\
\hline Dry matter & 93.6 & 93.5 & 93.6 & 93.9 & 93.7 & 93.3 \\
\hline Organic matter, $\%$ & 91.8 & 91.7 & 91.2 & 93.1 & 92.8 & 92.2 \\
\hline Gross energy, $\mathrm{MJ} \mathrm{kg}^{-1}$ & 18.0 & 18.1 & 18.1 & 16.8 & 16.7 & 16.6 \\
\hline Crude protein, $\%$ & 15.7 & 17.8 & 18.7 & 14.1 & 14.5 & 14.7 \\
\hline Ether extract, \% & 4.1 & 4.0 & 4.0 & 3.7 & 3.8 & 3.8 \\
\hline Ash, $\%$ & 8.2 & 8.3 & 8.8 & 6.9 & 7.2 & 7.8 \\
\hline Neutral detergent fiber, \% & 37.8 & 34.2 & 31.4 & 36.8 & 32.6 & 29.3 \\
\hline Acid detergent fiber, $\%$ & 15.6 & 14.0 & 12.0 & 19.8 & 17.5 & 14.8 \\
\hline $\mathrm{Ca}, \%$ & 0.5 & 0.5 & 0.5 & 0.3 & 0.5 & 0.6 \\
\hline $\mathrm{P}, \%$ & 0.2 & 0.3 & 0.3 & 0.2 & 0.2 & 0.3 \\
\hline $\begin{array}{l}\mathrm{C} 30=\text { diet containing } 30 \% \text { of concent } \\
\text { concentrate; Concentrates were purcl } \\
\text { Corporation, Beijing, China), which } \\
\text { limestone and salt. The nutrient conten } \\
\mathrm{P}=0.4-1.0 \%, \mathrm{NaCl}=0.5-2.0 \% \text {, Lysine }\end{array}$ & ude & orr & bra & oybean & $\begin{array}{l}- \text { an } \\
\text { ln Bre } \\
\text { lcium } \\
\text { ger }\end{array}$ & $\begin{array}{l}\text { ig } 50 \% \text { o } \\
\text { chnology } \\
\text { hosphate } \\
0.5-1.5 \%\end{array}$ \\
\hline
\end{tabular}

\subsection{Enteric Methane Emission Measurement}

Enteric $\mathrm{CH}_{4}$ emissions were measured from individual cows using the $\mathrm{SF}_{6}$ tracer technique with minor modification of Deighton et al. [9]. Generally, empty permeation tubes were filled with $450 \mathrm{~mL}$ of $99.999 \%$ pure $\mathrm{SF}_{6}$ by immersing in liquid nitrogen. The release rate was determined by incubating the permeation tubes in an oven at $39{ }^{\circ} \mathrm{C}$ and weighting each one twice a week for 4 weeks. The calculated releasing rate of the $\mathrm{SF}_{6}$ tubes ranged from 3.13 to 3.84 (mean, $3.28 \pm 0.175$ ) $\mathrm{mg} /$ day in period 1, from 3.10 to $3.70(3.32 \pm 0.266) \mathrm{mg} /$ day in period 2, and from 3.10 to $3.60(3.20 \pm 0.167) \mathrm{mg} /$ day in period 3, respectively. Each cow was randomly administrated with one $\mathrm{SF}_{6}$ tube using a balling gun three weeks before the commence of the experiment.

A back-mounted harness was used to support the canister (volume $=1.85 \mathrm{~L}$ ) for continuously sample collection; the canisters were washed by flushing $99.999 \%$ pure nitrogen and evacuated to over $98 \mathrm{kPa}$ vacuum. The sampling rate of canister was approximately $0.25 \mathrm{~mL} / \mathrm{min}$ by crimping a stainless-steel capillary tube within the sampling tubing. Canisters were removed after $24 \mathrm{~h}$ and residual vacuum was recorded before addition of nitrogen gas. Background gas samples of $\mathrm{SF}_{6}$ and $\mathrm{CH}_{4}$ were also collected daily by using six additional canisters that were either placed on the back of animals or about $2.0 \mathrm{~m}$ above ground level of the experimental barn.

Gas samples were analyzed using a gas chromatography system (GC126, Shanghai Precision Instruments Co., Ltd., Shanghai, China) equipped with a flame-ionization detector (FID) and an electron-capture detector (ECD). The ECD operated at $300{ }^{\circ} \mathrm{C}$ with a molecular sieve $0.5 \mathrm{~nm}$ column and the FID at $150^{\circ} \mathrm{C}$ with a Porapack N 80-100 mesh column (Shanghai Precision Instruments Co., Ltd., Shanghai, China) for determination of $\mathrm{SF}_{6}$ and $\mathrm{CH}_{4}$, respectively. Ultra-high purity nitrogen gas $(99.999 \%)$ was used as carrier gas at $40 \mathrm{~mL} / \mathrm{min}$ flow and analysis was performed after calibration with standard gases for $\mathrm{SF}_{6}$ and $\mathrm{CH}_{4}$. The daily $\mathrm{CH}_{4}$ emission was calculated as follows:

$$
\mathrm{CH}_{4}=\mathrm{SF}_{6} \times\left[\left(\mathrm{CH}_{4 \text { sample }}-\mathrm{CH}_{4 \text { background }}\right) /\left(\mathrm{SF}_{6 \text { sample }}-\mathrm{SF}_{6 \text { background }}\right)\right] \times(16 / 146) \times 1000
$$

where $\mathrm{CH}_{4}$ is the calculated emission $(\mathrm{g} / \mathrm{d}) ; \mathrm{SF}_{6}$ is the measured releasing rate of each $\mathrm{SF}_{6}$ permeation tube (mg/day); the concentration of $\mathrm{CH}_{4}$ sample and $\mathrm{CH}_{4}$ background are expressed in ppm and 
concentration of $\mathrm{SF}_{6}$ sample and $\mathrm{SF}_{6}$ background in ppt; 6 and 146 are the molecular mass $(\mathrm{g} / \mathrm{mol})$ of $\mathrm{CH}_{4}$ and $\mathrm{SF}_{6}$, respectively; the factor of 1,000 is used to calculate $\mathrm{CH}_{4}$ in units of $\mathrm{g} /$ day.

\subsection{Nutrient Digestibility and Laboratory Analyses}

During the last $6 \mathrm{~d}$ of each experimental period (digestibility experiment), 5 cows out of each treatment were moved to metabolic stalls for nutrient digestibility measurement using a modified method of acid-insoluble ash (AIA) [10]. Generally, rectal feces were collected from the rectum to obtain representative samples (day 1: 1000 and 2200 h; day 2: 0200 and 1400 h; day 3: 0500 and 1700 h; day 4: 0800 and 2000 h; day 5: 1100 and 2300 h; day 6: 0600 and 1800 h). Fresh samples over the 6 days period from each cow were composited and analyzed by using $2 \mathrm{~N} \mathrm{HCl}$. The equation used to calculate digestibility was as follows:

Nutrient digestibility $=100-[100 \times($ ADIA in DM consumed, $\% /$ ADIA in feces, $\%) /($ nutrient in feces, $\% /$ nutrient in consumed DM, \%)]

in which ADIA = acid detergent insoluble ash.

Representative feed samples were collected during adaptation and experimental period for chemical composition determinations. Dietary gross energy (GE) content was determined by bomb calorimetry (1108 Oxygen bomb, Parr Instruments, Moline, IL, USA). Dry matter, neutral detergent fiber (NDF), acid detergent fiber (ADF), crude fat, and ash were determined using AOAC International (2006), and crude protein (CP) was measured using combustion analyzer (Leco FP-528 N, Fullerton, CA, USA).

\subsection{Statistical Analyses}

The effect of dietary concentration levels on growth performance, nutrient digestibility and enteric $\mathrm{CH}_{4}$ emissions was evaluated using two analytical approaches as described by Dong et al. [1]. Generally, the ANOVA procedure was used with the three treatments fitted as a fixed effect and animals within each treatment fitted as random effects during the analysis. Other necessary variables such as initial BW and date of birth were fitted as covariates, when appropriate, for evaluation of enteric $\mathrm{CH}_{4}$ emissions. Prediction equations were developed using restricted maximum likelihood model as treatments were fitted as a fixed effect. Significant effects were noted at $p<0.05$. The statistical program used in the current study was Genstat 14.2 (14th edition; Lawes Agricultural Trust, Rothamsted, UK).

\section{Results}

\subsection{Effects on Nutrient Intake and Growth Performance}

Dietary ingredients and chemical composition are presented in Table 1 . The diets are planned to differ in concentrate contents and feed analysis indicated that NDF and ADF decreased with increasing concentrate feed contents in any of periods one to three. Accordingly, the opposite happened with the NFC fraction of the diets.

Nutrients and energy intake and growth performance are presented in Table 2. Overall, DM, OM, NFC, and GE intake increased with increasing concentrate contents in any of periods one to three $(p<0.05)$; however, these values did not differ significantly between the C40 and C50 treatments in period one or between C30 and C40 treatments in period two $(p>0.05)$. Dietary NDF intake was similar among the three treatments in period one ( 2.12 vs. 2.21 vs. 2.17 for C $30, C 40$ and C50 treatment, respectively, $p>0.05)$, whereas heifers in C30 treatments consumed more NDF than the other two treatments in periods two and three $(p<0.05)$. Weight gain increased with increasing concentrate feed contents in the diet $(p<0.05)$ with an average ADG value of $1.26,1.16$, and $0.97 \mathrm{~kg} /$ day for heifers in periods one to three, respectively. 
Table 2. Effects of different dietary concentrate levels on the growth performance of Holstein heifers at age of 9,12 and 15 months.

\begin{tabular}{|c|c|c|c|c|c|}
\hline \multirow{2}{*}{ Item } & \multicolumn{3}{|c|}{ Treatments } & \multirow{2}{*}{ SEM } & \multirow{2}{*}{$p$-Value } \\
\hline & C30 & $\mathrm{C} 40$ & C50 & & \\
\hline \multicolumn{6}{|c|}{9 months } \\
\hline Age, month & 9.5 & 9.5 & 9.4 & 1.46 & 0.987 \\
\hline BW, kg & $246.2^{\mathrm{b}}$ & $274.3^{\mathrm{a}}$ & $282.7^{a}$ & 5.16 & 0.046 \\
\hline DM intake, $\mathrm{kg} /$ day & $5.61^{\mathrm{b}}$ & $6.47^{\mathrm{a}}$ & $6.90^{\mathrm{a}}$ & 0.164 & $<0.01$ \\
\hline OM intake, kg/day & $5.15^{\mathrm{b}}$ & $5.94^{\mathrm{a}}$ & $6.30^{\mathrm{a}}$ & 0.152 & 0.007 \\
\hline $\mathrm{NDF}$ intake, $\mathrm{kg} /$ day & 2.12 & 2.21 & 2.17 & 0.093 & 0.07 \\
\hline NFC intake, kg/day & $1.92^{\mathrm{b}}$ & $2.31^{\mathrm{a}}$ & $2.57^{\mathrm{a}}$ & 0.041 & 0.024 \\
\hline GE intake, MJ/day & $100.8^{\mathrm{b}}$ & $117.4^{\mathrm{a}}$ & $124.9^{\mathrm{a}}$ & 2.74 & $<0.01$ \\
\hline ADG, kg/day & $1.10^{\mathrm{b}}$ & $1.33^{\mathrm{a}}$ & $1.36^{\mathrm{a}}$ & 0.085 & 0.448 \\
\hline \multicolumn{6}{|c|}{12 months } \\
\hline Age, month & 11.7 & 11.6 & 12.4 & 0.72 & 0.477 \\
\hline $\mathrm{BW}, \mathrm{kg}$ & $326.8^{\mathrm{b}}$ & $336.0^{\mathrm{b}}$ & $363.6^{\mathrm{a}}$ & 10.27 & 0.036 \\
\hline DM intake, $\mathrm{kg}$ /day & $6.98^{\mathrm{b}}$ & $7.06^{\mathrm{b}}$ & $7.18^{\mathrm{a}}$ & 0.233 & 0.001 \\
\hline OM intake, $\mathrm{kg} /$ day & $6.50^{\mathrm{b}}$ & $6.56^{\mathrm{b}}$ & $6.62^{\mathrm{a}}$ & 0.227 & 0.017 \\
\hline NDF intake, kg/day & $2.57^{\mathrm{a}}$ & $2.30^{\mathrm{b}}$ & $2.10^{\mathrm{b}}$ & 0.100 & 0.038 \\
\hline NFC intake, kg/day & $2.69^{\mathrm{b}}$ & $2.96^{\mathrm{b}}$ & $3.19^{\mathrm{a}}$ & 0.106 & 0.001 \\
\hline GE intake, MJ/day & $115.9^{\mathrm{b}}$ & $117.9^{b}$ & $124.6^{\mathrm{a}}$ & 0.500 & $<0.01$ \\
\hline ADG, kg/day & $0.97^{\mathrm{b}}$ & $1.14^{\mathrm{b}}$ & $1.39^{\mathrm{a}}$ & 0.062 & 0.010 \\
\hline \multicolumn{6}{|c|}{15 months } \\
\hline Age, month & 14.7 & 14.6 & 14.9 & 0.33 & 0.965 \\
\hline BW, kg & $402.2^{\mathrm{b}}$ & $424.4^{\mathrm{a}}$ & $429.3^{\mathrm{a}}$ & 8.82 & 0.945 \\
\hline DM intake, $\mathrm{kg} /$ day & $7.44^{\mathrm{C}}$ & $7.78^{\mathrm{b}}$ & $7.96^{\mathrm{a}}$ & 0.234 & 0.014 \\
\hline OM intake, $\mathrm{kg} /$ day & $6.86^{\mathrm{c}}$ & $7.22^{\mathrm{b}}$ & $7.42^{\mathrm{a}}$ & 0.126 & $<0.01$ \\
\hline NDF intake, $\mathrm{kg} /$ day & $2.93^{\mathrm{a}}$ & $2.53^{\mathrm{b}}$ & $2.18^{\mathrm{c}}$ & 0.228 & 0.031 \\
\hline NFC intake, $\mathrm{kg} /$ day & $3.06^{\mathrm{b}}$ & $3.26^{\mathrm{a}}$ & $3.30^{\mathrm{a}}$ & 0.179 & $<0.01$ \\
\hline GE intake, MJ/day & $124.9^{c}$ & $129.9^{b}$ & $132.2^{\mathrm{a}}$ & 1.69 & $<0.01$ \\
\hline $\mathrm{ADG}, \mathrm{kg} /$ day & $0.87^{\mathrm{b}}$ & $0.99^{\mathrm{a}}$ & $1.05^{\mathrm{a}}$ & 0.026 & 0.005 \\
\hline
\end{tabular}

$\mathrm{BW}=$ body weight, $\mathrm{OM}=$ organic matter, $\mathrm{NDF}=$ neutral detergent fiber, $\mathrm{ADF}=$ acid detergent fiber, $\mathrm{NFC}=$ non-fibrous carbohydrate, GE $=$ gross energy, $\mathrm{ADG}=$ average daily gain, $\mathrm{C} 30=$ diet containing $30 \%$ of concentrate, $\mathrm{C} 40=$ diet containing $40 \%$ of concentrate, $\mathrm{C} 50=$ diet containing $50 \%$ of concentrate, SEM $=$ standard error of means. ${ }^{a}, b, c$ values within a row with different superscripts differ significantly at $p<0.05$.

\subsection{Effects on Apparent Nutrient Digestibility}

Apparent nutrient digestibility data are presented in Table 3. Overall, CP and NDF digestibility increased with increasing concentrate feed contents throughout the three experimental periods $(p<0.05)$. However, DM and OM digestibility remained similar among the three treatments in period one, whereas both values increased linearly as concentrate increased in periods two and three $(p<0.05)$. Dietary ADF digestibility was unaffected by different concentrate contents in period two with a value of 68.4, 69.4 and 71.1 for C30, C40 and C50 treatments, respectively $(p>0.05)$. Moreover, there was an increasing trend in digestibility of average DM (74.47 vs. 75.50 vs. 78.47$)$, OM (76.43 vs. 79.20 vs. 80.50$)$ and ADF (68.42 vs. 69.63 vs. 74.00 ) for period one to three, respectively. 
Table 3. Effects of different dietary concentration level on apparent nutrient digestibility of Holstein cows at age of 9,12 and 15 months.

\begin{tabular}{|c|c|c|c|c|c|}
\hline \multirow{2}{*}{ Item } & \multicolumn{3}{|c|}{ Treatments } & \multirow{2}{*}{ SEM } & \multirow{2}{*}{$p$-Value } \\
\hline & C30 & $\mathrm{C} 40$ & C50 & & \\
\hline \multicolumn{6}{|c|}{9 months } \\
\hline Dry matter & 73.3 & 74.8 & 75.3 & 1.00 & 0.710 \\
\hline Organic matter & 75.3 & 76.8 & 77.2 & 1.00 & 0.740 \\
\hline Crude protein & $65.6^{c}$ & $69.3^{\mathrm{b}}$ & $76.5^{\mathrm{a}}$ & 1.53 & 0.003 \\
\hline Neutral detergent fiber & $69.2^{c}$ & $72.4^{\mathrm{b}}$ & $76.9^{a}$ & 1.52 & 0.012 \\
\hline Acid detergent fiber & $63.0^{c}$ & $69.4^{\mathrm{b}}$ & $72.9^{\mathrm{a}}$ & 1.87 & 0.041 \\
\hline \multicolumn{6}{|c|}{12 months } \\
\hline Dry matter & $73.5^{b}$ & $74.7^{\mathrm{b}}$ & $78.3^{\mathrm{a}}$ & 0.96 & 0.017 \\
\hline Organic matter & $77.1^{\mathrm{b}}$ & $78.6^{\mathrm{b}}$ & $81.9^{\mathrm{a}}$ & 0.92 & 0.047 \\
\hline Crude protein & $65.6^{b}$ & $69.3^{\mathrm{b}}$ & $76.5^{\mathrm{a}}$ & 1.53 & 0.002 \\
\hline Neutral detergent fiber & $60.8^{b}$ & $65.4^{\mathrm{a}}$ & $66.9^{\mathrm{a}}$ & 1.66 & 0.029 \\
\hline Acid detergent fiber & 68.4 & 69.4 & 71.1 & 1.46 & 0.141 \\
\hline \multicolumn{6}{|c|}{15 months } \\
\hline Dry matter & $75.7^{\mathrm{b}}$ & $76.5^{\mathrm{b}}$ & $83.2^{\mathrm{a}}$ & 1.04 & $<0.01$ \\
\hline Organic matter & $77.9^{\mathrm{b}}$ & $78.7^{\mathrm{b}}$ & $84.9^{\mathrm{a}}$ & 0.99 & $<0.01$ \\
\hline Crude protein & $72.7^{b}$ & $72.2^{\mathrm{b}}$ & $79.6^{\mathrm{a}}$ & 1.10 & $<0.01$ \\
\hline Neutral detergent fiber & $73.1^{\mathrm{b}}$ & $75.4^{\mathrm{a}}$ & $76.7^{\mathrm{a}}$ & 0.92 & 0.028 \\
\hline Acid detergent fiber & $71.3^{\mathrm{b}}$ & $73.5^{\mathrm{b}}$ & $77.2^{\mathrm{a}}$ & 1.11 & 0.046 \\
\hline
\end{tabular}

C30 = diet containing $30 \%$ of concentrate; $\mathrm{C} 40=$ diet containing $40 \%$ of concentrate; $\mathrm{C} 50=$ diet containing $50 \%$ of concentrate, $\mathrm{SEM}=$ standard error of means. ${ }^{\mathrm{a}, \mathrm{b}, \mathrm{c}}$ values within a row with different superscripts differ significantly at $p<0.05$.

\subsection{Effects on Enteric $\mathrm{CH}_{4}$ Emission}

Enteric $\mathrm{CH}_{4}$ emission data of each experiment period are presented in Table 4. Daily $\mathrm{CH}_{4}$ production and $\mathrm{CH}_{4}-\mathrm{E}$ were significantly affected by treatments that both sets of parameters decreased linearly with increasing concentrate feed contents in the diets in any periods of one to three $(p<0.05)$.

Table 4. Effects of different dietary concentration level on enteric methane $\left(\mathrm{CH}_{4}\right)$ emissions of Holstein cows at age of 9,12 and 15 months.

\begin{tabular}{|c|c|c|c|c|c|}
\hline \multirow{2}{*}{ Item } & \multicolumn{3}{|c|}{ Treatments } & \multirow{2}{*}{ SEM } & \multirow{2}{*}{$p$-Value } \\
\hline & $\mathrm{C} 30$ & $\mathrm{C} 40$ & C50 & & \\
\hline \multicolumn{6}{|c|}{9 months } \\
\hline $\mathrm{CH}_{4}, \mathrm{~g} /$ day & $114.90^{\mathrm{a}}$ & $107.10^{b}$ & $93.66^{\mathrm{c}}$ & 2.584 & $<0.01$ \\
\hline $\mathrm{CH}_{4} / \mathrm{MBW}, \mathrm{g} / \mathrm{kg}^{0.75}$ & $1.68^{\mathrm{a}}$ & $1.59^{\mathrm{b}}$ & $1.42^{\mathrm{C}}$ & 0.031 & 0.002 \\
\hline $\mathrm{CH}_{4} / \mathrm{DM}$ intake, $\mathrm{g} / \mathrm{kg}$ & $20.57^{\mathrm{a}}$ & $16.56^{\mathrm{b}}$ & $13.57^{\mathrm{C}}$ & 0.643 & $<0.01$ \\
\hline $\mathrm{CH}_{4} / \mathrm{OM}$ intake, $\mathrm{g} / \mathrm{kg}$ & $26.15^{\mathrm{a}}$ & $21.39^{\mathrm{b}}$ & $17.15^{\mathrm{c}}$ & 0.734 & $<0.01$ \\
\hline $\mathrm{CH}_{4} / \mathrm{NDF}$ intake, $\mathrm{g} / \mathrm{kg}$ & $60.66^{\mathrm{a}}$ & $47.55^{\mathrm{b}}$ & $35.40^{c}$ & 2.99 & $<0.01$ \\
\hline $\mathrm{CH}_{4}-\mathrm{E}, \mathrm{MJ} /$ day & $6.40^{\mathrm{a}}$ & $5.96^{\mathrm{b}}$ & $5.21^{\mathrm{c}}$ & 0.131 & $<0.01$ \\
\hline $\mathrm{CH}_{4}$-E/GE intake & $0.0686^{\mathrm{a}}$ & $0.0552^{\mathrm{b}}$ & $0.0454^{\mathrm{c}}$ & 0.00264 & $<0.01$ \\
\hline \multicolumn{6}{|c|}{12 months } \\
\hline $\mathrm{CH}_{4}, \mathrm{~g} /$ day & $159.68^{\mathrm{a}}$ & $133.16^{b}$ & $119.32^{c}$ & 5.054 & $<0.01$ \\
\hline $\mathrm{CH}_{4} / \mathrm{MBW}, \mathrm{gkg}^{0.75}$ & $2.09^{\mathrm{a}}$ & $1.71^{\mathrm{b}}$ & $1.50^{\mathrm{c}}$ & 0.079 & 0.001 \\
\hline $\mathrm{CH}_{4} / \mathrm{DM}$ intake, $\mathrm{g} / \mathrm{kg}$ & $22.88^{\mathrm{a}}$ & $18.85^{\mathrm{b}}$ & $16.63^{c}$ & 0.903 & $<0.01$ \\
\hline $\mathrm{CH}_{4} / \mathrm{OM}$ intake, $\mathrm{g} / \mathrm{kg}$ & $27.13^{\mathrm{a}}$ & $22.65^{\mathrm{b}}$ & $20.40^{c}$ & 0.958 & $<0.01$ \\
\hline $\mathrm{CH}_{4} / \mathrm{NDF}$ intake, $\mathrm{g} / \mathrm{kg}$ & $63.77^{a}$ & $55.81^{\mathrm{b}}$ & $54.60^{\mathrm{b}}$ & 1.744 & 0.019 \\
\hline $\mathrm{CH}_{4}-\mathrm{E}, \mathrm{MJ} / \mathrm{day}^{-1}$ & $7.89^{\mathrm{a}}$ & $7.41^{\mathrm{b}}$ & $6.64^{\mathrm{c}}$ & 0.281 & $<0.001$ \\
\hline $\mathrm{CH}_{4}$-E/GE intake & $0.0742^{\mathrm{a}}$ & $0.0618^{\mathrm{b}}$ & $0.0558^{\mathrm{b}}$ & 0.00321 & $<0.001$ \\
\hline
\end{tabular}


Table 4. Cont.

\begin{tabular}{|c|c|c|c|c|c|}
\hline \multirow{2}{*}{ Item } & \multicolumn{3}{|c|}{ Treatments } & \multirow{2}{*}{ SEM } & \multirow{2}{*}{$p$-Value } \\
\hline & $\mathrm{C} 30$ & $\mathrm{C} 40$ & $\mathrm{C} 50$ & & \\
\hline \multicolumn{6}{|c|}{15 months } \\
\hline $\mathrm{CH}_{4}, \mathrm{~g} /$ day & $219.58^{a}$ & $214.86^{b}$ & $193.77^{c}$ & 4.17 & $<0.01$ \\
\hline $\mathrm{CH}_{4} / \mathrm{MBW}, \mathrm{g} / \mathrm{kg}^{0.75}$ & $2.39^{\mathrm{a}}$ & $2.26^{\mathrm{a}}$ & $2.02^{\mathrm{b}}$ & 0.058 & 0.013 \\
\hline $\mathrm{CH}_{4} / \mathrm{DM}$ intake, $\mathrm{g} / \mathrm{kg}$ & $23.17^{\mathrm{a}}$ & $19.94^{\mathrm{b}}$ & $16.92^{\mathrm{c}}$ & 0.776 & $<0.01$ \\
\hline $\mathrm{CH}_{4} / \mathrm{OM}$ intake, $\mathrm{g} / \mathrm{kg}$ & $24.95^{\mathrm{a}}$ & $21.54^{\mathrm{b}}$ & $18.20^{\mathrm{c}}$ & 0.837 & $<0.01$ \\
\hline $\mathrm{CH}_{4} / \mathrm{NDF}$ intake, $\mathrm{g} / \mathrm{kg}$ & $69.39^{\mathrm{a}}$ & $67.12^{\mathrm{b}}$ & $64.83^{c}$ & 1.312 & 0.039 \\
\hline $\mathrm{CH}_{4}-\mathrm{E}, \mathrm{MJ} /$ day & $12.77^{\mathrm{a}}$ & $11.96^{\mathrm{b}}$ & $10.78^{\mathrm{c}}$ & 0.232 & $<0.01$ \\
\hline $\mathrm{CH}_{4}$-E/GE intake & $0.0769^{a}$ & $0.0665^{\mathrm{b}}$ & $0.0568^{c}$ & 0.00374 & $<0.01$ \\
\hline
\end{tabular}

$\mathrm{MBW}=$ metabolic body weight, $\mathrm{DM}=$ dry matter, $\mathrm{OM}=$ organic matter, NDF = neutral detergent fiber, $\mathrm{CH}_{4}-\mathrm{E}=$ methane energy, $\mathrm{GE}=$ gross energy, $\mathrm{C} 30=$ diet containing $30 \%$ of concentrate; $\mathrm{C} 40=$ diet containing $40 \%$ of concentrate; $\mathrm{C} 50=$ diet containing $50 \%$ of concentrate, $\mathrm{SEM}=$ standard error of means. ${ }^{\mathrm{a}, \mathrm{b}, \mathrm{c}}$ values within a row with different superscripts differ significantly at $p<0.05$.

Individual $\mathrm{CH}_{4}$ intensity including $\mathrm{CH}_{4} / \mathrm{DM}$ intake, $\mathrm{CH}_{4} / \mathrm{OM}$ intake, and $\mathrm{CH}_{4} / \mathrm{NDF}$ intake decreased linearly with increasing dietary concentrate feed contents throughout the three experiment periods, whereas no difference was observed for $\mathrm{CH}_{4} / \mathrm{NDF}$ intake between $\mathrm{C} 40$ and $\mathrm{C} 50$ (55.81 vs. $54.60 \mathrm{~g} / \mathrm{kg})$ treatments in period two $(p>0.05) . \mathrm{CH}_{4}$-E per gross energy intake $\left(\mathrm{Y}_{\mathrm{m}}\right)$ decreased significantly $(p<0.05)$ with increasing concentrate contents in any periods of one to three. Furthermore, although comparison of the effect of experimental periods on $\mathrm{CH}_{4}$ emissions was the objective of this study, the average of $Y_{m}$ value was $0.0564,0.0639$, and 0.0667 in periods one to three, respectively.

\subsection{Development of Prediction Equations}

Prediction equations of $\mathrm{CH}_{4}$ emissions in each period are presented in Tables 4 and 5. Growth and feed intake parameters were used to develop these relationships, which were significantly correlated $(p<0.01)$ with coefficient of determination values ranging from 0.27 to 0.74 .

Table 5. Prediction equations of methane $\left(\mathrm{CH}_{4}\right)$ emission for Holstein heifers at age of 9,12 , and 15 months.

\begin{tabular}{ccccc}
\hline Item & Equations & $\mathrm{SE}$ & $\mathbf{R}^{\mathbf{2}}$ & Eq. \\
\hline $\mathrm{CH}_{4}$ & $=0.13_{(0.106)} \times \mathrm{BW}+68.6_{(29.15)}$ & 0.330 & 0.47 & $(1)$ \\
& $=24.21_{(1.133)} \times \mathrm{DM}$ intake $-51_{(7.34)}$ & 0.999 & 0.67 & $(2)$ \\
$\mathrm{CH}_{4}-\mathrm{E}$ & $=0.08_{(0.004)} \times \mathrm{GE}$ intake $-2.72_{(0.467)}$ & 0.999 & 0.69 & $(3)$ \\
$\mathrm{CH}_{4}$ & $=0.19_{(0.151)} \times \mathrm{BW}+78.6_{(49.64)}$ & 0.461 & 0.42 & $(4)$ \\
& $=36.27_{(6.712)} \times \mathrm{DM}$ intake $-88_{(12.24)}$ & 0.782 & 0.71 & $(5)$ \\
$\mathrm{CH}_{4}-\mathrm{E}$ & $=0.11_{(0.012)} \times \mathrm{GE}$ intake $-4.65_{(1.785)}$ & 0.766 & 0.72 & $(6)$ \\
$\mathrm{CH}_{4}$ & $=0.29_{(0.161)} \times \mathrm{BW}+84.9_{(68.61)}$ & 0.461 & 0.46 & $(7)$ \\
$\mathrm{CH}_{4}-\mathrm{E}$ & $=51.72_{(4.640)} \times \mathrm{DM}$ intake $-193.9_{(22.49)}$ & 0.979 & 0.74 & $(8)$ \\
\hline
\end{tabular}

$\mathrm{CH}_{4}-\mathrm{E}=$ methane energy (MJ/day), $\mathrm{BW}=$ body weight $(\mathrm{kg}), \mathrm{DM}=$ dry matter (kg/day), $\mathrm{GE}$ = gross energy $(\mathrm{MJ} /$ day); $\mathrm{SE}=$ standard error

Overall, relationships obtained in period three had highest values of determination values when compared with those from the other two periods. The strongest relationship was observed between $\mathrm{CH}_{4}$ emission and DM intake in period three (Equation (8) in Table 5, $\mathrm{R}^{2}=0.74$ ), whereas $\mathrm{CH}_{4}$ production was relatively poor related with BW for heifers in period 1 (Equation (1) in Table 5, $R^{2}=0.47$ ). Furthermore, emissions data derived from all three periods were pooled to develop overall $\mathrm{CH}_{4}$ prediction equations (Figures 1 and 2). Feed intake and BW were significantly correlated with $\mathrm{CH}_{4}$ emission $(p<0.01)$ and coefficient of determination value was 0.727 and 0.802 for linear and non-linear equations, respectively. Furthermore, a range of linear and non-linear prediction models 
were developed using the whole data sets of animal production and feed intake values (Equations (10) to (20), Table 6). Generally, improved values of $R^{2}$ can be observed with more variables were incorporated in to the models. For example, highest value of $R^{2}$ of 0.820 was observed for the models relating $\mathrm{CH}_{4}$-E to $\mathrm{BW}$, DM intake, and NFC intake $(p<0.01)$, whereas a relatively low value of $\mathrm{R}^{2}$ of 0.593 was observed for Equation (12), which relates $\mathrm{CH}_{4}$-E to DM intake and NDF intake $(p<0.01)$. However, there was no such trends for non-linear models as highest value of $\mathrm{R}^{2}$ was observed for the relationship between $\mathrm{CH}_{4}$-E and NDF intake (Equation (18)).

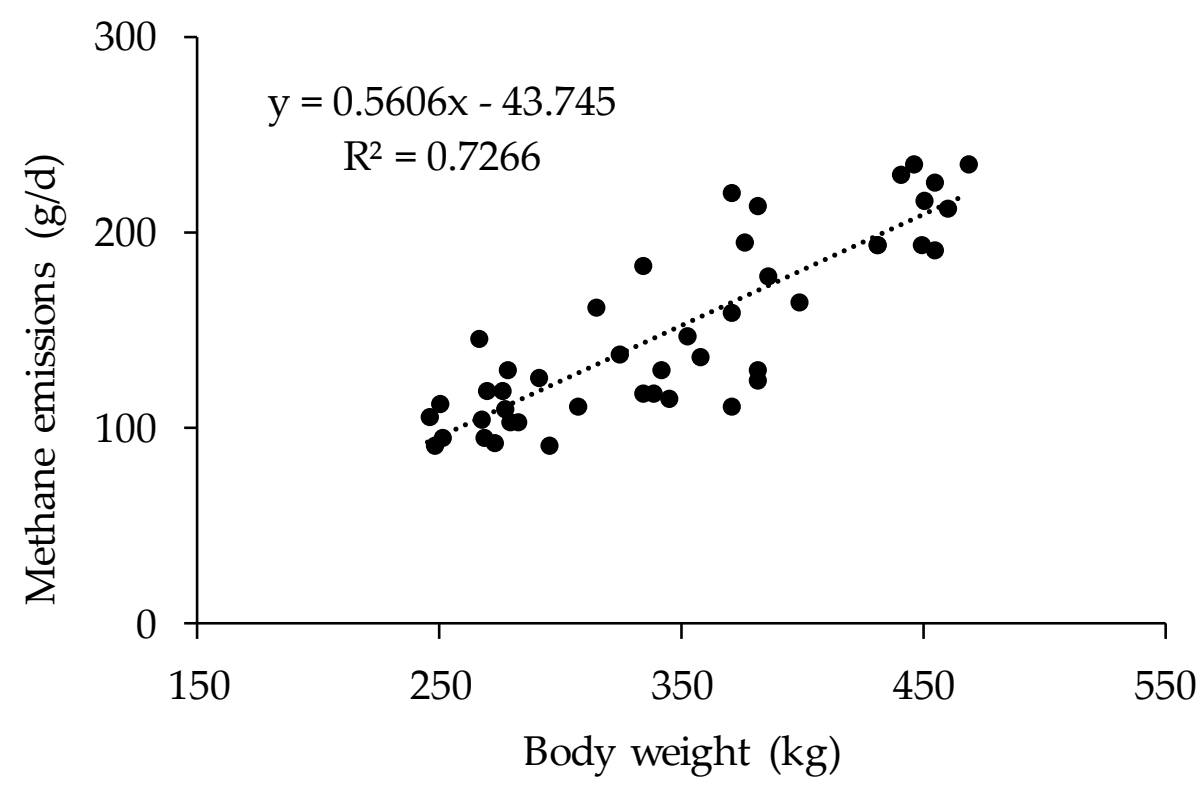

Figure 1. Linear relationship between body weight and enteric methane emissions of Holstein heifers.

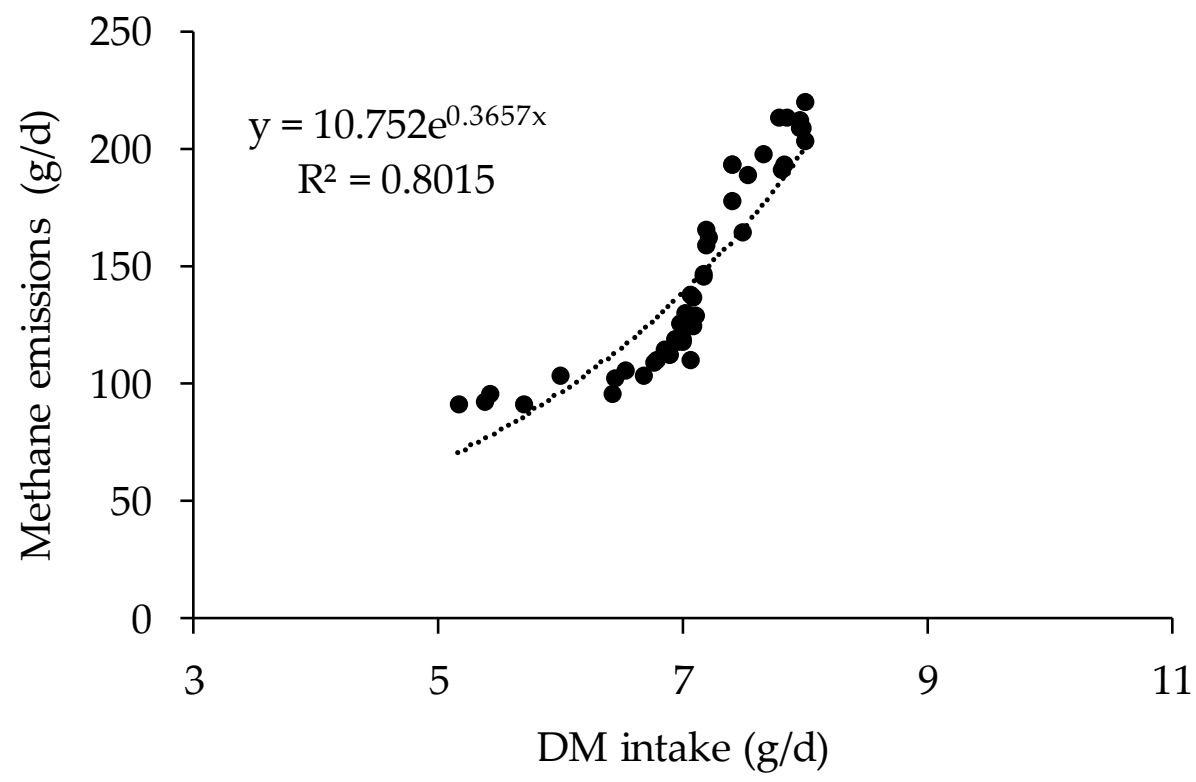

Figure 2. Non-linear relationship between dry matter intake and enteric methane emissions of Holstein heifers. 
Table 6. Development of methane prediction models for Holstein heifers using the whole data sets.

\begin{tabular}{|c|c|c|c|c|}
\hline Item $^{1}$ & Equations & SE & $\mathbf{R}^{2}$ & Eq. \\
\hline \multirow{6}{*}{$\mathrm{CH}_{4}-\mathrm{E}(\mathrm{MJ} /$ day $)$} & Linear models & & & \\
\hline & $\begin{array}{c}0.026(0.0043) \times \mathrm{BW}(\mathrm{kg})+0.69(0.431) \times \mathrm{DM} \text { intake }(\mathrm{kg} / \mathrm{day})- \\
5.564(1.1940)\end{array}$ & 0.337 & 0.742 & $(10)$ \\
\hline & $\begin{array}{c}3.18(0.408) \times \mathrm{DM} \text { intake }(\mathrm{kg} / \text { day })+1.74(0.598) \times \mathrm{NDF} \text { intake } \\
(\mathrm{kg} / \text { day })-9.426(2.6003)\end{array}$ & 0.682 & 0.593 & $(11)$ \\
\hline & $\begin{array}{c}1.75(0.399) \times \mathrm{DM} \text { intake }(\mathrm{kg} / \mathrm{d})-2.71(0.648) \times \mathrm{NFC} \text { intake } \\
(\mathrm{kg} / \text { day })-8.552(2.4150)\end{array}$ & 0.549 & 0.655 & $(12)$ \\
\hline & $\begin{array}{c}0.024(0.0041) \times \mathrm{BW}(\mathrm{kg})+1.22(0.456) \times \mathrm{DM} \text { intake }(\mathrm{kg} / \text { day })+ \\
1.15(0.459) \times \mathrm{NDF} \text { intake }(\mathrm{kg} / \text { day })-5.389(2.0681)\end{array}$ & 0.261 & 0.777 & $(13)$ \\
\hline & $\begin{array}{c}0.023(0.0037) \times \mathrm{BW}(\mathrm{kg})+0.28(0.038) \times \mathrm{DM} \text { intake }(\mathrm{kg} / \text { day })- \\
2.04(0.486) \times \mathrm{NFC} \text { intake }(\mathrm{kg} / \text { day })-4.872(1.8634) \\
\text { Non-linear models }\end{array}$ & 0.132 & 0.820 & $(14)$ \\
\hline \multirow{6}{*}{$\mathrm{CH}_{4}-\mathrm{E}(\mathrm{MJ} /$ day $)$} & $5.564(1.1206) \times \exp (0.0276(0.0037) \times \mathrm{DM}$ intake $(\mathrm{kg} /$ day $))$ & 0.396 & 0.461 & $(15)$ \\
\hline & $4.333(1.0177) \times \mathrm{DM}$ intake $(\mathrm{kg} / \text { day })^{0.232(0.0452)}$ & 0.601 & 0.446 & (16) \\
\hline & $2.465(0.7452) \times \exp (0.0075(0.0008) \times$ NDF intake $(\mathrm{kg} / \mathrm{d}))$ & 0.452 & 0.411 & (17) \\
\hline & $2.204(0.6514) \times$ NDF intake $(\mathrm{kg} / \text { day })^{0.084(0.0072)}$ & 0.377 & 0.489 & (18) \\
\hline & $0.926(0.0452) \times \exp (0.0672(0.00121) \times$ NFC intake $(\mathrm{kg} /$ day $))$ & 0.514 & 0.385 & (19) \\
\hline & $0.527(0.0271) \times \mathrm{NDF}$ intake $(\mathrm{kg} / \text { day })^{0.541(0.0362)}$ & 0.602 & 0.434 & (20) \\
\hline
\end{tabular}

${ }^{1} \mathrm{CH}_{4}-\mathrm{E}=$ methane energy, $\mathrm{BW}=$ body weight, $\mathrm{DM}=$ dry matter, $\mathrm{NDF}=$ neutral detergent fiber, $\mathrm{NFC}=$ non-fibrous carbohydrate; $\mathrm{SE}=$ standard error.

\section{Discussion}

\subsection{Effects on Feed Intake and Growth Performance}

A number of studies have demonstrated that increasing dietary concentrate contents would increase feed intake of heifers, although they are typically fed high-fiber diets due to physiological and economic considerations [11]. In accordance with the previous studies, moving from 2.09 to $3.59 \mathrm{~kg} / \mathrm{day}$ and 2.23 to $3.98 \mathrm{~kg} /$ day from of concentrate treatments increased DM intake by 0.20 and $0.52 \mathrm{~kg} /$ day in period two and three, respectively. Aguerre at al. [12] reported a significant increase of NDF intake from 5.4 to $6.5 \mathrm{~kg} /$ day as dietary forage-to-concentrate increased from 47:53 to 68:32. However, although only a numerical change was observed in NDF intake, NFC intake increased significantly as concentrate contents increased from 30 to $50 \%$ in period one. In the current study, alfalfa was introduced into the diets in the last two periods. This high-quality forage would be responsible for the significant increase of feed intake for the C50 treatments due to its good palatability and high level of digestibility [13]. Consequently, increased ADG were achieved in the current study as a direct result of higher energy density of the higher concentrate diets and increased feed intake [14].

\subsection{Effects on Apparent Nutrient Digestibility}

Generally, nutrient digestibility increased with increased concentrate supplementation for heifers in any periods of the present study. Moody et al. [15] reported that increasing dietary corn silage contents reduced DM digestibility of Holstein heifers either at the age of 6 or 12 months. Jiao et al. [16] found similar digestibility values of DM, NDF and ADF to our results for heifers at various ages. Different from our findings, Moody et al. [15] reported that NDF digestibility decreased as concentrate proportion increased in the diet, and concluded that this significant reduction of NDF digestibility may be due to the variations of passage of different forage in the diet and growth condition of animals [17]. However, as corn silage was commonly used in each experimental period, only alfalfa was introduced into the period two and three of the present study. This inclusion of different forage types might explain different nutrient digestibility among the three treatments in any period of one to three. Sarwar et al. [18] reported little difference of nitrogen digestibility for Holstein cows fed diets varying in proportion of NDF. Recent studies conducted by Drewnoski and Poore [19] and Trotta et al. [20] found that increasing dietary concentrate level increased the total tract CP digestibility from 53.1 to $58.1 \%$ for beef cattle. The current study showed that there was a positive relationship between 
dietary concentrate level and CP digestibility, which had similar trends to the digestibility values of other nutrients. Nousiainene et al. [21] suggested that increased CP digestibility was associated with improved diet digestibility, which may be resulted from increased dietary CP concentrate and a dilution of metabolic and endogenous fecal nitrogen. However, these authors also suggested that the amount of dietary CP concentration instead of amount of concentrate was related to changes of dietary $\mathrm{CP}$ digestibility. Dietary CP content increased from 15.7 to $18.7 \%$ (period 1) or from 14.1 to $14.7 \%$ (period 2) with concentrate level increasing from 30 to $50 \%$ in the present study. However, it still needs further study to elucidate the direct relationship between nutrient digestibility and dietary composition as some cofounding factors need to be considered during analysis. However, it is worth noting that the nutrient digestibility values in the present study were obtained using acid-insoluble ash as internal marker, as this method has been extensively recognized for determination of diet digestibility due to reliable digestibility estimates [22,23]. Previously, the standard procedure for measuring total-tract apparent digestibility involved total collection of feces and urine, whereas alternative approaches including acid-insoluble ash and indigestible NDF were proposed and used as they required a small number of animals and produced accurate results [24,25]. Nevertheless, nutrient digestibility values obtained in the present study were consistent with the previous studies, which indicated that acid-insoluble ash method may be a suitable and convenient method although the total collection should still be considered the best choice [26].

\subsection{Effects on Enteric Methane Emissions}

Due to the large population of China's dairy industries, it is becoming increasingly important to quantify $\mathrm{CH}_{4}$ emissions for cows at different ages and under various production systems. However, until recently, several studies investigated the effects of dietary concentrate contents on $\mathrm{CH}_{4}$ emissions for heifers. Boland et al. [27] reported similar $\mathrm{CH}_{4}$ emissions (121 vs. $132 \mathrm{~g} /$ day) for grazing beef heifer that consumed different herbage masses. Jiao et al. [16] examined $\mathrm{CH}_{4}$ emissions from heifer and steer at various growth stage, and reported an average daily $\mathrm{CH}_{4}$ emission of 93.5, 159.5, 175.0, and $188.5 \mathrm{~g} /$ day for young stock at the age of $6,12,18$, and 22 months under confined condition. These values are similar to the recent study of Morrison et al. [28] who measured $\mathrm{CH}_{4}$ emissions from grazing heifers using the $\mathrm{SF}_{6}$ tracer technique. However, emission data for heifers at the age of 15 months were higher than those for confined heifer and steer or for young stock in grazing condition [28].

As enteric $\mathrm{CH}_{4}$ emission represents the final production of ruminal fermentation via methanogenesis, it can be significantly affected by a range of factors including animal physiological state, dietary components, and measurement technique. Increased concentrate proportion resulted in reduced $\mathrm{CH}_{4}$ emissions in the current study. These values were consistent with studies of Muñoz et al. [29], who also decreased $\mathrm{CH}_{4}$ production with increasing concentrate level up to 6 and $5 \mathrm{~kg}$ of concentrate per day, respectively. Generally, inclusion of high level of concentrate in the diet represents higher content of readily fermentable substance (e.g., starch) than that of high forage diets. Previous studies demonstrated that starch-rich diets reduced ruminal $\mathrm{pH}$ and $\mathrm{H}_{2}$ concentration, and shifted fermentation patter towards to an increased propionate formation, which would depress the activity of methanogens and consequently reduce $\mathrm{CH}_{4}$ emissions [30,31]. Moreover, the composition and structure of ruminal methanogens was demonstrated to differ across heifer physiological stages, which would affect the enteric $\mathrm{CH}_{4}$ emissions of heifers [32]. Although the main objective of the current was not to examine the archaeal community in the rumen, results showed an increasing trend in $\mathrm{CH}_{4}$ emissions as the growth of heifer advanced, which would reflect the changes and distribution of ruminal methanogens.

Regional or national enteric $\mathrm{CH}_{4}$ emission inventories in many countries are currently estimated using the Tier-2 methodology from International Panel on Climate Change (IPCC) guidelines. As with Tier-2 approaches, default prediction values for $Y_{m}$ from adult dairy cows in the 1997 (0.060) [33] and 2006 (0.065) [5] IPCC guidelines were recommended for the $\mathrm{CH}_{4}$ estimation of the whole dairy population. However, adoption of a default and fixed value has becoming a major concern because it can vary considerably with varying geographic conditions, cow breed and physiological stages, and 
dietary characteristics [6,7]. Boadi et al. [34] reported an $Y_{m}$ value of 0.067 or 0.076 for yearling heifers either fed ad-libitum or under restricted feeding condition. Morrison et al. [28] calculated $Y_{m}$ values for calves, yearling heifer, and in-calf heifer with an average age of 8.5, 14.5 and 20.5 months, and found that the calculated $Y_{m}$ was $0.057,0.0675,0.059$ for each period. In accordance with those results from confined lactating cows or heifers at pasture, a range of $Y_{m}$ values between 0.0454 and 0.0769 were obtained across all heifer ages in the current study, which lied within the range (0.036-0.114) obtained under diverse production systems [35]. Therefore, these prediction factors achieved on a regional production basis in China can be used and improve the prediction accuracy for cows at specific developmental stages. Furthermore, variations of $Y_{m}$ values were also examined when heifers were fed different concentrate contents in the diet. As report previously, variations of dietary components such as starch: NDF ratios can change the rumen fermentation environment and methanogenesis functions, which consequently affect the $\mathrm{CH}_{4}$ emissions. In the present study, $\mathrm{Y}_{\mathrm{m}}$ values decreased from 0.0686 to $0.0454,0.0742$ to $0.0558,0.0769$ to 0.0568 when concentrate intake increased from 1.68 to $3.45,2.09$ to $3.59,2.23$ to $3.98 \mathrm{~kg} /$ day in period 1 to 3, respectively. These results are consistent with the grazing studies of van Wyngaard et al. [35], who reported that $Y_{m}$ of lactating Jersey cows significantly decreased from 0.0891 to 0.0785 when the concentrate increased from 0 to $8 \mathrm{~kg} /$ day.

\subsection{Prediction Equations for Enteric Methane Emissions}

Enteric $\mathrm{CH}_{4}$ emission predictions have been widely developed based on mathematical or statistical association of nutrient intake, dietary nutrient composition and digestibility and other animal factors with enteric $\mathrm{CH}_{4}$ emissions [36]. In agreement with previous studies, DM and GE intake were the best predictors of $\mathrm{CH}_{4}$ emissions in this study with values of $\mathrm{R}^{2}$ ranging from 0.67 to 0.74 . Similar $\mathrm{R}^{2}$ values of 0.68 with DM intake and 0.70 with GE intake were reported for beef cattle measured using respiration calorimeters [37]. Appuhamy et al. [7] evaluated performance of more than 40 empirical models in predicting enteric $\mathrm{CH}_{4}$ emissions, and suggested that DM intake alone may be sufficient to achieve satisfactory prediction accuracy inventory purposes [38]. A meta-analysis conducted by Charmley et al. [39] showed that a large data set including both dairy and beef cattle can significantly enhance the relationship between DM intake and $\mathrm{CH}_{4}$ emissions, with a high value of determination coefficient and an intercept close to zero when DM intake ranged from 2 to $28 \mathrm{~kg} / \mathrm{day}$. The data was pooled together and a linear or nonlinear relationship was observed between BW, DM intake and $\mathrm{CH}_{4}$ emissions in the current study. However, curvilinear relationship between DM intake and $\mathrm{CH}_{4}$ production was observed when dairy cows were fed relatively high proportion of concentrate [40]. It was suggested that linear relationship between $\mathrm{DM}$ intake and $\mathrm{CH}_{4}$ production can be achieved when the concentrate level was below 30\% [39]. Yan et al. [37] reported that the coefficient of determination for the relationship between DM intake and $\mathrm{CH}_{4}$ emissions were highly affected by several factors including growth stage, dietary concentrations of protein and carbohydrate fractions. For example, coefficients for DM intake increased from 24.21 to 51.72 for heifers at the age of 9 to 15 months, although this difference did not reach significance. Moreover, lower values of $R^{2}$ from 0.42 to 0.47 were observed when animal characteristic such as BW was used as a single predictor variable. Jiao et al. [16] reported an increase of $0.252 \mathrm{~kg} /$ day $\mathrm{CH}_{4}$ for an increase of $1 \mathrm{~kg}$ of heifer $\mathrm{BW}$, which was similar to our findings that an average increase of $0.203 \mathrm{~kg} / \mathrm{day} \mathrm{CH}_{4}$ was observed for each unit increase of BW.

Although linear models can be mathematically developed using dietary intake and composition variables, enteric $\mathrm{CH}_{4}$ emissions may not follow a linear trend as generation of $\mathrm{CH}_{4}$ can be affected by ruminal function and fermentation dynamics. Among the non-linear models developed using the whole data sets of the present study, a highest $\mathrm{R}^{2}$ value of 0.82 was observed when BW, DM intake and NFC intake were incorporate in to the equation. However, a range of relative lower values of $R^{2}$ were also found for those exponential or power equations. This result was in consistent with the previous research of Mills et al. [41] and Patra et al. [42], who found minor difference in RMSE percentage between the linear and non-linear models. Although non-linear models required more variables to obtained the accurate methane emissions results, Mills et al. [41] suggested that 
non-linear models would be better for quantifying $\mathrm{CH}_{4}$ production in a wide range of production variables; especially, as they could be more appropriate when extreme values were obtained during the practical application [42]. The slopes of dietary DM and NDF intake were positively related to enteric $\mathrm{CH}_{4}$ emissions, whereas increasing dietary NFC intake may reduce $\mathrm{CH}_{4}$ emission. Diets rich in non-structural carbohydrates such as starch and sugars are converted to propionate in the rumen with less hydrogen and $\mathrm{CH}_{4}$ production. However, fermentation of fibrous materials would favour the formation of acetate and butyrate, which would have positive impact on $\mathrm{CH}_{4}$ emissions.

\section{Conclusions}

It is concluded that increasing dietary concentrate contents improves feed intake and growth performance, and nutrient digestibility. Enteric methane emissions decrease significantly with increasing concentrate contents. A range of $\mathrm{CH}_{4}$ conversion factors are derived from the current study, reflecting the variations of animal and dietary characteristics under the typical production regimes in China. Together with prediction equations, these data will be of great importance for development of regional or national emission inventories and mitigation approaches for heifers at specific growth stage.

Author Contributions: Conceptualization, Q.D.; methodology, L.D.; data curation, B.L.; writing-original draft preparation, L.D.; writing—review and editing, Q.D.; visualization, L.D.; supervision, Q.D.; project administration, Q.D.; funding acquisition, L.D. and Q.D.

Funding: This research was funded by Program of International S\&T Cooperation (2016YFE0109000), National Science Foundation for Young Scientists of China (31802085), National Key Research and Development Program of China (2017YFF0211702 and 2017YFF0211701), and Young Scientist Lifting Project (2017-2019).

Acknowledgments: The authors thank colleagues in Feed Research Institute and Century Love International Investment Group Co. Ltd., Beijing for providing kind assistance in animal experiments, sample processing, and data collection. The authors would like to thank reviewers for their suggestions and comments on this work.

Conflicts of Interest: The authors declare no conflict of interest.

\section{References}

1. Dong, L.F.; Ferris, C.P.; McDowell, D.A.; Yan, T. Effects of diet forage proportion on maintenance energy requirement and the efficiency of metabolizable energy use for lactation by lactating dairy cows. J. Dairy Sci. 2015, 98, 1-10. [CrossRef] [PubMed]

2. Second National Communication on Climate Change of China (SNCCCC). National Development and Reform Commission of the People's Republic of China. Available online: http://www.cchina.gov.cn/archiver/ cchinaen/UpFile/Files/Default/20130218145208096785.pdf/ (accessed on 2 December 2015).

3. Zhu, G.D.; Ma, X.Y.; Gao, Z.L.; Ma, W.Q.; Li, J.G.; Cai, Z.J. Characterizing $\mathrm{CH}_{4}$ and $\mathrm{N}_{2} \mathrm{O}$ emissions from an intensive dairy operation in summer and fall in China. Atmos. Environ. 2014, 38, 245-253. [CrossRef]

4. Yang, Y.; Zhang, J.J.; Wang, C. Forecasting China's Carbon Intensity-Is China on Track to Comply with Its Copenhagen Commitment? Energy J. 2018, 39, 63-86. [CrossRef]

5. Intergovernmental Panel on Climate Change (IPCC). 2006 IPCC Guidelines for National Greenhouse Gas Inventories. In Prepared by the National Greenhouse Gas Inventories Programme; Eggleston, H.S., Buendia, L., Miwa, K., Ngara, T., Tanabe, K., Eds.; IGES: Hayama, Japan, 2006.

6. Bannink, A.; van Schijndel, M.W.; Dijkstra, J. A model of enteric fermentation in dairy cows to estimate methane emission for the Dutch National Inventory Report using the IPCC Tier 3 approach. Anim. Feed Sci. Technol. 2011, 166, 603-618. [CrossRef]

7. Appuhamy, J.A.D.R.N.; France, J.; Kebreab, E. Models for predicting enteric methane emissions from dairy cows in North America, Europe, and Australia and New Zealand. Glob. Chang. Biol. 2016, 22, 3039-3056. [CrossRef]

8. Xue, B.; Wang, L.Z.; Yan, T. Methane emission inventories for enteric fermentation and manure management of yak, buffalo and dairy and beef cattle in China from 1988 to 2009. Agric. Ecosyst. Environ. 2014, 195, 202-210. [CrossRef] 
9. Deighton, M.H.; Williams, S.R.O.; Hannah, M.C.; Eckard, R.J.; Boland, T.M.; Wales, W.J.; Moate, P.J. A modified sulphur hexafluoride tracer technique enables accurate determination of enteric methane emissions from ruminants. Anim. Feed Sci. Technol. 2014, 197, 47-63. [CrossRef]

10. Van Keulen, J.; Young, B.A. Evaluation of acid-insoluble ash as a natural marker in ruminant digestibility studies. J. Anim. Sci. 1997, 44, 282-287. [CrossRef]

11. Kljak, K.; Heinrichs, B.S.; Heinrichs, A.J. Fecal particle dry matter and fiber distribution of heifers fed ad libitum and restricted with low and high forage quality. J. Dairy Sci. 2019, 102, 4694-4703. [CrossRef]

12. Aguerre, M.J.; Wattiaux, M.A.; Powell, J.M.; Broderick, G.A.; Arndt, C. Effect of forage-to-concentrate ratio in dairy cow diets on emission of methane, carbon dioxide, and ammonia, lactation performance, and manure excretion. J. Dairy Sci. 2011, 94, 3081-3093. [CrossRef]

13. Kljak, K.; Pino, F.; Heinrichs, A.J. Effect of forage to concentrate ratio with sorghum silage as a source of forage on rumen fermentation, $\mathrm{N}$ balance, and purine derivative excretion in limit-fed dairy heifers. J. Dairy Sci. 2017, 100, 213-223. [CrossRef]

14. Reynolds, C.K.; Tyrrell, H.F.; Reynolds, P.J. Effects of diet forage-to-concentrate ratio and intake on energy metabolism in growing beef heifers: Net nutrient metabolism by visceral tissues. J. Nutr. 1991, 121, 1004-1015. [CrossRef]

15. Moody, M.L.; Zanton, G.I.; Daubert, J.M.; Heinrichs, A.J. Nutrient utilization of differing forage-to-concentrate ratios by growing Holstein heifers. J. Dairy Sci. 2007, 90, 5580-5586. [CrossRef] [PubMed]

16. Jiao, H.P.; Dale, A.J.; Carson, A.F.; Murray, S.; Gordon, A.W.; Ferris, C.P. Effect of concentrate feed level on methane emissions from grazing dairy cows. J. Dairy Sci. 2014, 97, 7043-7053. [CrossRef]

17. Nakamura, T.; Owen, F.G. High amounts of soyhulls for pelleted concentrate diets. J. Dairy Sci. 1989, 72, 988-994. [CrossRef]

18. Sarwar, M.; Firkins, J.L.; Eastridge, M.L. Effects of varying forage and concentrate carbohydrates on nutrient digestibilities and milk production by dairy cows. J. Dairy Sci. 1992, 75, 1533-1542. [CrossRef]

19. Drewnoski, M.E.; Poore, M.H. Effect of supplementation frequency on ruminal fermentation and digestion by steers fed medium-quality hay and supplemented with a soybean hull and corn gluten feed blend. J. Anim. Sci. 2012, 90, 881-891. [CrossRef]

20. Trotta, R.J.; Klotz, J.L.; Harmon, D.L. Effects of source and level of dietary energy supplementation on in vitro digestibility and methane production from tall fescue-based diets. Anim. Feed Sci. Technol. 2018, 242, 41-47. [CrossRef]

21. Nousiainen, J.; Rinne, M.; Huhtanen, P. A meta-analysis of feed digestion in dairy cows. 1. The effects of forage and concentrate factors on total diet digestibility. J. Dairy Sci. 2009, 92, 5019-5030. [CrossRef]

22. Huhtanen, P.; Kaustell, K.; Jaakkola, S. The use of internal markers to predict total digestibility and duodenal flow of nutrients in cattle given six different diets. Anim. Feed Sci. Technol. 1994, 48, 211-227. [CrossRef]

23. Rice, E.M.; Aragona, K.M.; Moreland, S.C.; Erickson, P.S. Supplementation of sodium butyrate to postweaned heifer diets: Effects on growth performance, nutrient digestibility, and health. J. Dairy Sci. 2019, 102, 1-10. [CrossRef]

24. Nikkhah, A.; Alikhani, M.; Amanlou, H. Effects of Feeding Ground or Steam-Flaked Broom Sorghum and Ground Barley on Performance of Dairy Cows in Midlactation. J. Dairy Sci. 2004, 87, 122-130. [CrossRef]

25. Lee, C.; Hristov, A.N. Evaluation of acid-insoluble ash and indigestible neutral detergent fiber as total-tract digestibility markers in dairy cows fed corn silage-based diets. J. Dairy Sci. 2013, 96, 5295-5299. [CrossRef]

26. De Marco, M.; Miraglia, N.; Peiretti, P.G.; Bergero, D. Apparent digestibility of wheat bran and extruded flax in horses determined from the total collection of feces and acid-insoluble ash as an internal marker. Animal 2012, 6, 227-231. [CrossRef]

27. Boland, T.M.; Quinlan, C.; Pierce, K.M.; Lynch, M.B.; Kenny, D.A.; Kelly, A.K.; Purcell, P.J. The effect of pasture pregrazing herbage mass on methane emissions, ruminal fermentation, and average daily gain of grazing beef heifers. J. Anim. Sci. 2013, 91, 3867-3874. [CrossRef]

28. Morrison, S.J.; McBride, J.; Gordon, A.W.; Wylie, A.R.G.; Yan, T. Methane Emissions from Grazing Holstein-Friesian Heifers at Different Ages Estimated Using the Sulfur Hexafluoride Tracer Technique. Eng. 2017, 3, 753-759. [CrossRef]

29. Muñoz, C.; Hube, S.; Morales, J.M.; Yan, T.; Ungerfeld, E.M. Effects of concentrate supplementation on enteric methane emissions and milk production of grazing dairy cows. Livest. Sci. 2015, 175, 37-46. [CrossRef] 
30. Zhang, X.M.; Wang, M.; Wang, R.; Ma, Z.Y.; Long, D.L.; Mao, H.X.; Wen, J.N.; Bernard, L.A.; Beauchemin, K.A.; Tan, Z.L. Urea plus nitrate pretreatment of rice and wheat straws enhances degradation and reduced methane production in in vitro ruminal culture. J. Sci. Food Agric. 2018, 98, 5205-5211. [CrossRef]

31. Lan, W.; Yang, C.L. Ruminal methane production: Associated microorganisms and the potential of applying hydrogen-utilizing bacteria for mitigation. Sci. Total Environ. 2019, 654, 1270-1283. [CrossRef]

32. Cunha, C.S.; Marcondes, M.I.; Veloso, C.M.; Mantovani, H.C.; Pereira, L.G.R.; Tomich, T.R.; Dill-McFarland, K.A.; Suen, G. Compositional and structural dynamics of the ruminal microbiota in dairy heifers and its relationship to methane production. J. Sci. Food Agric. 2019, 99, 210-218. [CrossRef]

33. Intergovernmental Panel on Climate Change (IPCC). Revised 1996 IPCC Guidelines for National Greenhouse Gas Inventories; Intergovernmental Panel on Climate Change, IPCC/OECD/IEA: Bracknell, UK, 1997.

34. Boadi, D.A.; Wittenberg, K.M. Methane production from dairy and beef heifers fed forages differing in nutrient density using the sulphur hexafluoride $\left(\mathrm{SF}_{6}\right)$ tracer gas technique. Can. J. Anim. Sci. 2011, 82, 201-206. [CrossRef]

35. Van Wyngaard, J.D.V.; Meeske, R.; Erasmus, L.J. Effect of concentrate level on enteric methane emissions, production performance, and rumen fermentation of Jersey cows grazing kikuyu-dominant pasture during summer. J. Dairy Sci. 2018, 101, 1-13. [CrossRef]

36. Hristov, A.N.; Kebreab, E.; Niu, M.; Oh, J.; Bannink, A.; Bayat, A.R.; Boland, T.B.; Brito, A.F.; Casper, D.P.; Crompton, L.A.; et al. Uncertainties in enteric methane inventories, measurement techniques, and prediction models. J. Dairy Sci. 2018, 101, 1-20. [CrossRef]

37. Yan, T.; Porter, M.G.; Mayne, C.S. Prediction of methane emission from beef cattle using data measured in indirect open-circuit respiration calorimeters. Animal 2009, 3, 1455-1462. [CrossRef]

38. Hess, P.S.A.; Eckard, R.J.; Jacobs, J.L.; Hannah, M.C.; Moate, P.J. Comparison of five methods for the estimation of methane production from vented in vitro systems. J. Sci. Food Agric. 2019, 99, 109-116. [CrossRef]

39. Charmley, E.; Williams, S.R.O.; Moate, P.J.; Hegarty, R.S.; Herd, R.M.; Oddy, V.H.; Reyenga, P.; Staunton, K.M.; Anderson, A.; Hannah, M.C. A universal equation to predict methane production of forage-fed cattle in Australia. Anim. Prod. Sci. 2016, 56, 169-180. [CrossRef]

40. Knapp, J.R.; Laur, G.L.; Vadas, P.A.; Weiss, W.P.; Tricarico, J.M. Enteric methane in dairy cattle production: Quantifying the opportunities and impact of reducing emissions. J. Dairy Sci. 2014, 97, 3231-3261. [CrossRef]

41. Mills, J.A.N.; Kebreab, E.; Yates, C.M.; Crompton, L.A.; Cammell, S.B.; Dhanoa, M.S.; Agnew, R.E.; France, J. Alternative approaches to predicting methane emissions from dairy cows. J. Anim. Sci. 2003, 81, 3141-3150. [CrossRef]

42. Patra, A.K.; Lalhriatpuii, M. Development of statistical models for prediction of enteric methane emission from goats using nutrient composition and intake variables. Agric. Ecosyst. Environ. 2016, 215, 89-99. [CrossRef]

(C) 2019 by the authors. Licensee MDPI, Basel, Switzerland. This article is an open access article distributed under the terms and conditions of the Creative Commons Attribution (CC BY) license (http://creativecommons.org/licenses/by/4.0/). 